\title{
Estimación de la función de utilidad del consumidor ecuatoriano en el año 2012
}

\author{
Miguel Cervantes - Jiménez ${ }^{1}$, Eduardo León- Castañeda² \\ Fecha de recepción: 8 de Diciembre 2017 \\ Fecha de aceptación: 28 de Marzo 2018
}

\section{Resumen}

El presente artículo genera tres funciones de utilidad del consumidor ecuatoriano en los ámbitos nacional, urbano y rural con base en una función de utilidad tipo Cobb-Douglas y datos de la Encuesta Nacional de Ingresos y Gastos de Hogares Urbanos y Rurales 2011-2012, cada una con doce bienes cuyos exponentes representan la proporción del gasto de cada bien respecto al gasto total. Los consumidores del Ecuador destinan la mitad de gasto en alimentos y bebidas no alcohólicas, transporte y otros bienes y servicios. El gasto destinado a los alimentos en las zonas urbanas y rurales es de 23 por ciento y 32 por ciento, respectivamente. El decil I destina el 60 por ciento de su ingreso a satisfacer estas necesidades, mientras que el decil $X$ utiliza entre el 41 y el 50 por ciento de sus recursos, dependiendo de si habitan zonas urbanas o rurales, respectivamente. Las funciones de utilidad de los consumidores se pueden utilizar para analizar los efectos de la aplicación de políticas públicas como el establecimiento de precios controlados, subsidios o de cambios impositivos.

Palabras clave: Preferencias, función de utilidad del consumidor, función Cobb-Douglas, consumo.

\section{Abstract}

This article generates three consumer utility functions at the national, urban and rural level based on a Cobb-Douglas type utility function and data from the National Survey of Income and Expenditures of Urban and Rural Households 2011-2012, each with twelve goods; exponents represent the proportion of the expenditure of each good regarding the total expense. Consumers in Ecuador spend half on food and non-alcoholic beverages, transport and other goods and services. Spending on food in urban and rural areas is 23 percent and 32 percent, respectively. The decile I allocate 60 percent of its income to satisfy these needs, while the decile $X$ uses between 41 and 50 percent of its resources, depending on whether they inhabit urban or rural areas, respectively. The consumer utility functions can be used to analyze the effects of the application of public policies such as the establishment of controlled prices, subsidies or tax changes.

Keywords: Preferences, consumer utility function, Cobb-Douglas function, consumption.

\section{Introducción}

El gasto en consumo final de los hogares es la variable agregada más importante de las economías en el mundo, ya que ocupa entre el sesenta y ochenta por ciento de la producción total. En el caso de la economía ecuatoriana entre los años 2010 y 2016, según cifras del Banco Central del Ecuador, el PIB creció 3,4 por ciento en promedio cada año, en tanto el consumo de los hogares creció en promedio anual al 1,8 por ciento, lo que incidió para que la proporción del consumo de los hogares respecto del Producto Interno Bruto (PIB) bajara de 65 por ciento a 59 por ciento del año 2010 al 2016; en contraste, el gasto de consumo final del gobierno ascendió de trece a quince por ciento en el mismo periodo.

El objetivo del presente artículo es generar tres funciones de utilidad del consumidor ecuatoriano en los ámbitos nacional, urbano y rural con base en una función tipo Cobb-Douglas y datos provenientes de la Encuesta Nacional de Ingresos y Gastos de Hogares Urbanos y Rurales 2011-2012, cada una con doce bienes.

El artículo se estructura en dos apartados, en el primero, se presenta la metodología fundamentada en la elección racional de los consumidores de la escuela neoclásica utilizando una función de utilidad del consumidor tipo Cobb-Douglas y, en el segundo, se exhiben los resultados de la construcción de la función de utilidad del consumidor ecuatoriano en los ámbitos nacional, urbano y rural utilizando los datos de la encuesta señalada. Al final se incluyen las conclusiones.

\section{Metodología}

Para identificar las jerarquizaciones de las preferencias del consumidor, la teoría neoclásica desarrolló las funciones de utilidad. Para Cervantes (2014, pág. 65), las "funciones de utilidad son una forma matemática de describir el sistema de preferencia", en donde lo importante es el orden y no el valor que asume la función, ya que no existe la medida de "útiles".

Las preferencias de los consumidores se construyen a partir de axiomas, a saber:

Completitud: dadas dos canastas de consumo $\left(\mathrm{x}_{1}, \mathrm{x}_{2}\right)$ y $\left(\mathrm{y}_{1}, \mathrm{y}_{2}\right)$, si $\left(\mathrm{x}_{1}, \mathrm{x}_{2}\right)$ $\geq\left(\mathrm{y}_{1}, \mathrm{y}_{2}\right)$ ó $\left(\mathrm{y}_{1}, \mathrm{y}_{2}\right) \geq\left(\mathrm{x}_{1}, \mathrm{x}_{2}\right)$ ó las dos situaciones, entonces el consumidor es indiferente a cualquiera de las dos canastas $\left(\mathrm{x}_{1}, \mathrm{x}_{2}\right) \sim\left(\mathrm{y}_{1}, \mathrm{y}_{2}\right)$. Lo importante de este axioma radica en que el consumidor puede identificar y comparar todas las canastas de consumo.

- Reflexividad: cualquier canasta es al menos tan preferida como ella misma: $\left(x_{1}, x_{2}\right) \geq\left(x_{1}, x_{2}\right)$.

- Transitividad: Dadas tres canastas $\left(\mathrm{x}_{1}, \mathrm{x}_{2}\right),\left(\mathrm{y}_{1}, \mathrm{y}_{2}\right)$ y $\left(\mathrm{z}_{1}, \mathrm{z}_{2}\right)$ si $\left(\mathrm{x}_{1}, \mathrm{x}_{2}\right) \geq\left(\mathrm{y}_{1}, \mathrm{y}_{2}\right)$ y tenemos que $\left(\mathrm{y}_{1}, \mathrm{y}_{2}\right) \geq\left(\mathrm{z}_{1}, \mathrm{z}_{2}\right)$, entonces $\left(\mathrm{x}_{1}, \mathrm{x}_{2}\right) \geq\left(\mathrm{z}_{1}, \mathrm{z}_{2}\right)$.

Monotonía: si $\left(\mathrm{x}_{1}, \mathrm{x}_{2}\right) \sim\left(\mathrm{y}_{1}, \mathrm{y}_{2}\right)$ si a la canasta $\left(\mathrm{y}_{1}, \mathrm{y}_{2}\right)$ se le agrega una unidad de algún bien se tiene que $\left(\mathrm{y}_{1}+1, \mathrm{y}_{2}\right)>\left(\mathrm{x}_{1}, \mathrm{x}_{2}\right)$.

- Convexidad: cuando las canastas son convexas se prefieran las canastas medias a las extremas, si $\left(x_{1}, x_{2}\right) \sim\left(y_{1}, y_{2}\right)$ y la media ponderada es la canasta $\left(0.5 x_{1}+\right.$ $\left.0.5 y_{1}, 0.5 x_{2}+0.5 y_{2}\right)$, dicha canasta es al menos tan buena como los extremos.

A partir del sistema de preferencias se construyen las funciones de utilidad, las que deben cumplir las siguientes propiedades: la utilidad de la canasta $(x 1, x 2)$ será mayor que la utilidad de la canasta (y1, y2), si y sólo si, la canasta ( $x 1, x 2)$ es estrictamente preferida a la canasta (y1, y2), o sea, $u\left(x_{1}, x_{2}\right)>u\left(y_{1}, y_{2}\right) \Leftrightarrow\left(x_{1}, x_{2}\right) \succ\left(y_{1}, y_{2}\right)$ (Cervantes, 2014).

A pesar del reducido uso de este tipo de herramienta en la evaluación económica, se han realizado algunos esfuerzos, principalmente en el campo de los modelos de equilibrios general computables deterministas o estocásticos, en los que se ocupan funciones de utilidad a partir de las cuentas nacionales, constituyéndose en la principal función objetivo de un modelo para la evaluación de políticas públicas.

Se han explorado otras aplicaciones de la función de utilidad a través de los modelos de elección discreta con modelos logit y tobit, tal como se muestra en el libro Microeconometría y decisión de autoría de Cabrer, Sancho y Serrano (2001) y en Microeconometría de Arcarons y Ramírez (2012).

También se han utilizado con diferentes herramientas econométricas para la medición de los parámetros de funciones de utilidad, principalmente translogaritmicas y Cobb-Douglas. En este tenor, Holman (1998) estimó una función de utilidad con decisiones intertemporales, por lo cual aparece el consumo en bienes para periodos distintos y añade las tenencias de dinero como otro bien que genera utilidad, esta medición la realizó a través del método de momentos generalizados. Ewis y Fisher (1984) realizaron la estimación de la función de utilidad de la demanda de dinero para el caso de 
Estados Unidos a través de modelos de rezagos distribuidos. Christensen, Lau y Lawrence (1975) calcularon la función de utilidad translogaritmica y la función indirecta de utilidad para bienes duraderos y no duraderos, utilizando un modelo de sistema de demanda casi ideal (AIDS por sus siglas en inglés). Kim (1988) estimó la función de utilidad de la demanda educativa para el caso de Estados Unidos a través del modelo AIDS y Fortune (1979) estimó una función de utilidad translogaritmica para la medición de cuotas y tarifas.

La función de utilidad para el caso de Ecuador se construye con la disponibilidad de datos al año 2011-2012. Se utiliza la forma funcional tipo Cobb Douglas, la que cumple con las propiedades de monotonía, convexidad y continuidad, cuya expresión matemática es la siguiente:

$$
U=\prod_{i=1}^{n} x_{i}^{\alpha_{i}}
$$

Donde $\mathrm{x}$ representa la mercancía i-ésima, las cuales provienen de las encuestas de ingresos y gastos de hogares, el parámetro a corresponde a la proporción del gasto que se realiza en las mercancías respecto al gasto total, las cuales se obtienen de la encuesta mencionada.

Para obtener el parámetro alfa (a) se plantea el problema de maximización del consumo de las mercancías, considerando sólo dos resulta:

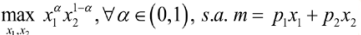

Para resolverlo se genera la función auxiliar lagrangiana: $\max _{\substack{1,1 \\ x_{1}}} x_{1}^{\alpha} x_{2}^{1-a}+\lambda\left(m-p_{1} x_{1}-p_{2} x_{2}\right)$ se obtienen las tres condiciones de primer orden y se despejan $\mathrm{x}_{1}$ y $\mathrm{x}_{2}$, con lo que se obtienen las funciones de demanda de cada una de las mercancías, esto es: $x_{1}=\alpha \frac{m}{p_{1}}$ y $x_{2}=(1-\alpha) \frac{m}{p_{2}}$ En el caso de la mercancía $x 1$ para obtener el parámetro (a) se procede a despejarla, con lo que se obtiene lo siguiente: $\alpha=\frac{p_{1} x_{1}}{m}$, lo que implica que el parámetro alfa es igual al gasto en la mercancía dividido por el ingreso.

\section{Resultados}

Con base en la función descrita en la metodología, la función Cobb-Douglas aplicada para el caso de Ecuador es la siguiente:

$$
U=\prod_{i=1}^{12} x_{i}^{\alpha_{i}}
$$

Donde $\mathrm{x}$ representa el número de mercancías incluidas en el modelo, las cuales provienen de la Encuesta Nacional de Ingresos y Gastos de Hogares Urbanos y Rurales (Instituto Nacional de Estadística y Censos, 2012) y el parámetro a es la proporción del gasto que realiza cada uno de los deciles en las mercancías consideradas.

Se calculan diversas funciones de utilidad para el caso de Ecuador, esto debido a la desagregación de la encuesta, de forma tal que se obtuvieron tres funciones de tipo Cobb-Douglas (total, zona urbana y zona rural) para cada uno de los diez deciles.

La tabla 1 presenta el gasto total realizado en cada una de las mercancías consideradas en el presente estudio. Como se puede observar, en Ecuador los tres principales rubros del gasto son: alimentos y bebidas no alcohólicas, transporte y bienes y servicios diversos; mientras que los rubros en los cuales es menor la proporción del gasto son: bebidas alcohólicas, tabaco y

\begin{tabular}{|c|c|c|c|c|}
\hline \multicolumn{5}{|c|}{$\begin{array}{l}\text { Tabla 1. Gasto corriente mensual } \\
\text { Ecuador } \\
2012\end{array}$} \\
\hline & Rubros de gasto & $\begin{array}{l}\text { Total de } \\
\text { hogares }\end{array}$ & Total de gasto & $\begin{array}{l}\text { Participación } \\
\text { porcentual en } \\
\text { el gasto total }\end{array}$ \\
\hline Total & & 3.923 .123 & $\$ 2.393 .571 .816$ & $100 \%$ \\
\hline 1. & Alimentos y bebidas no alcohólicas & 3.859 .422 & $\$ 584.496 .341$ & $24 \%$ \\
\hline 2. & $\begin{array}{l}\text { Bebidas alcohólicas, tabaco y } \\
\text { estupefacientes }\end{array}$ & 1.047 .175 & $\$ 17.303 .834$ & $1 \%$ \\
\hline 3. & Prendas de vestir y calzado & 3.746 .135 & $\$ 190.265 .816$ & $8 \%$ \\
\hline & $\begin{array}{l}\text { Alojamiento, agua, electricidad, gas y otros } \\
\text { combustibles }\end{array}$ & $3,839,376$ & $\$ 177.342 .239$ & $7 \%$ \\
\hline & $\begin{array}{l}\text { Muebles, articulos para el hogar y para la } \\
\text { conservación ordinaria del mismo }\end{array}$ & 3.894 .660 & $\$ 142.065 .518$ & $6 \%$ \\
\hline & Salud & 3568.938 & $\$ 179.090 .620$ & $7 \%$ \\
\hline & Transporte & 3599.702 & $\$ 349.497 .442$ & $15 \%$ \\
\hline & Comunicaciones & 3.222 .409 & $\$ 118,734,692$ & $5 \%$ \\
\hline & Recreación y cultura & 3.343 .612 & $\$ 109.284 .976$ & $5 \%$ \\
\hline & Educación & $1,058,751$ & $\$ 104.381 .478$ & $4 \%$ \\
\hline & Restaurantes y hoteles & 3.068 .688 & $\$ 184.727 .177$ & $8 \%$ \\
\hline & Bienes y servicios diversos & 3.885 .451 & $\$ 236.381 .682$ & $10 \%$ \\
\hline
\end{tabular}
estupefacientes.
Con base en la participación porcentual en el gasto total de la tabla 1, la función de utilidad para el caso de Ecuador es la siguiente:

$$
U_{\text {total }}=x_{1}^{0.24} x_{2}^{0.01} x_{3}^{0.08} x_{4}^{0.07} x_{5}^{0.06} x_{6}^{0.07} x_{7}^{0.15} x_{8}^{0.05} x_{9}^{0.05} x_{10}^{0.04} x_{11}^{0.08} x_{12}^{0.10}
$$

Donde "U" representa la función de utilidad y los valores " $x 1, x 2, x 3, \ldots, x 12$ ", representan los bienes y servicios que integran el nivel de gasto de los hogares. Sus exponentes representan la proporción del ingreso que es destinada al gasto de cada bien.

Esta función de utilidad indica que en Ecuador el 24 por ciento del gasto se destina al consumo de alimentos y bebidas no alcohólicas, posteriormente se ubica el gasto en transporte el cual tiene una ponderación de 15 por ciento y bienes y servicios con un 10 por ciento; en conjunto las tres mercancías representan el 49 por ciento del gasto que realizan los habitantes del país sudamericano.

Sin embargo, existen diferencias respecto de las zonas en las cuales se realiza el gasto, es por ello que se procedió a la construcción de dos funciones de utilidad adicionales, una para zonas urbanas y otra para rurales. Con base en la Encuesta Nacional de Ingresos y Gastos de Hogares Urbanos y Rurales 2011-2012 se presentan ambas funciones de utilidad:

$$
\begin{gathered}
U_{\text {urbana }}=x_{1}^{0.23} x_{2}^{0.01} x_{3}^{0.08} x_{4}^{0.08} x_{5}^{0.06} x_{6}^{0.07} x_{7}^{0.15} x_{8}^{0.05} x_{9}^{0.05} x_{10}^{0.05} x_{11}^{0.08} x_{12}^{0.10} \\
U_{\text {rural }}=x_{1}^{0.32} x_{2}^{0.01} x_{3}^{0.09} x_{4}^{0.04} x_{5}^{0.06} x_{6}^{0.08} x_{7}^{0.14} x_{8}^{0.04} x_{9}^{0.04} x_{10}^{0.02} x_{11}^{0.06} x_{12}^{0.10}
\end{gathered}
$$

Si bien los rubros principales en los que gastan son los mismos que en la función total, las ponderaciones difieren principalmente en el gasto en alimentos y bebidas, ya que mientras en las zonas urbanas el porcentaje destinado es de 23 por ciento, en las zonas rurales la proporción asciende a 32 por ciento, lo cual refleja que a medida que el ingreso de los individuos se incrementa, se puede gastar en otros bienes y servicios distintos a los alimentos incrementando el nivel de vida. El sector urbano gasta una mayor proporción que el sector rural en alojamiento, agua, luz y gas, y también en educación.

En la tabla 2 se desglosan los bienes y servicios por deciles y por tipo de zona en que habitan los hogares, además se exhibe la proporción del gasto en cada uno de los rubros. Las proporciones del gasto muestran que el decil I en la zona rural gasta el 40 por ciento de su ingreso en alimentos y bebidas contra el 42 por ciento que realiza el mismo decil en la zona urbana, lo que puede explicarse porque en las zonas rurales algunas de las familias se satisfacen con autoconsumo reduciendo el gasto en alimentos y bebidas. En cuanto al decil $X$, se observa que en las zonas rurales el gasto en bienes de consumo es el doble al que se realiza en las ciudades. Mientras que el decil VI en las zonas rurales gasta once puntos porcentuales más que en las zonas urbanas. En materia de transporte y otros bienes y servicios, los dos rubros de mayor ponderación, la participación entre los deciles dependiendo de la zona en que habitan ronda entre el 8 y 22 por ciento para transporte y entre 9 y 10 por ciento para otros bienes, no encontrándose diferencias significativas.

\begin{tabular}{|c|c|c|c|c|c|c|}
\hline \multirow[t]{2}{*}{ Área geográfica y división de gasto } & \multicolumn{3}{|c|}{ Urbana } & \multicolumn{3}{|c|}{ Rural } \\
\hline & Decill & $\begin{array}{c}\text { Decil } \\
\text { VI }\end{array}$ & Decil X & Decill & $\begin{array}{c}\text { Decil } \\
\text { VI }\end{array}$ & Decil X \\
\hline Gasto corriente de consumo & 1 & 1 & 1 & 1 & 1 & 1 \\
\hline Alimentos y bebidas no alcohólicas & 0,42 & 0,25 & 0,10 & 0,40 & 0,36 & 0,19 \\
\hline Bebidas alcoholicas, tabaco y estupefacientes & 0,01 & 0,01 & 0,01 & 0,01 & 0,01 & 0,01 \\
\hline Prendas de vestir y calzado & 0,07 & 0,08 & 0,07 & 0,11 & 0,08 & 0,08 \\
\hline $\begin{array}{l}\text { Alojamiento, agua, electricidad, gas y otros } \\
\text { combustibles }\end{array}$ & 0,09 & 0,09 & 0,07 & 0,05 & 0,05 & 0,04 \\
\hline $\begin{array}{l}\text { Muebles, artículos para el hogar y para la } \\
\text { conservadón ordinaria del mismo }\end{array}$ & 0,05 & 0,05 & 0,08 & 0,07 & 0,06 & 0,07 \\
\hline Salud & 0,05 & 0.07 & 0,08 & 0,06 & 0,09 & 0,09 \\
\hline Transporte & 0,08 & 0,13 & 0,21 & 0,10 & 0,12 & 0,22 \\
\hline Comunicaciones & 0,03 & 0,06 & 0,06 & 0,02 & 0,03 & 0,05 \\
\hline Recreación y cultura & 0,04 & 0,04 & 0,06 & 0,04 & 0,04 & 0,05 \\
\hline Educación & 0,01 & 0,04 & 0,07 & 0,01 & 0,02 & 0,04 \\
\hline Restaurantes $y$ hoteles & 0,05 & 0,09 & 0,08 & 0,04 & 0,06 & 0,08 \\
\hline Bienes y servicios diversos & 0,10 & 0,09 & 0,10 & 0,10 & 0,10 & 0,09 \\
\hline
\end{tabular}

Tabla 2. Estimación de la función de utilidad Cobb-Douglas por decil y tipo de zona habitada Ecuador 2012

En el caso del decil I el gasto realizado en los tres rubros de mayor ponderación es del 60 por ciento tanto en las zonas urbanas como rurales. Para el decil VI se observan diferencias significativas, en las zonas rurales la participación alcanza el 57 por ciento contra el 48 por ciento de los sectores 
urbanos, es decir, una diferencia de 9 puntos porcentuales. El decil $X$ de las zonas urbanas destina el 41 por ciento de sus recursos, en tanto las zonas rurales el 50 por ciento.

\section{Conclusiones}

Una de las herramientas teóricas más importantes en la ciencia económica es el uso de las funciones de utilidad del consumidor, representación matemática del sistema de preferencias de los individuos y que permiten jerarquizar el consumo de los agentes económicos.

Una de las complicaciones del análisis empírico de la teoría de la elección racional del consumidor es que se genera por medio de un sustento axiomático, y no es común obtenerlos por medio de la modelación estadística o econométrica. Sin embargo, se han realizado esfuerzos por llevar a la práctica el análisis de las funciones de utilidad de los consumidores, es el caso de los modelos de equilibrio general aplicado los cuales requieren de la estimación de dichas funciones para la solución de los problemas de optimización del consumo; las funciones más empleadas han sido las de elasticidad de sustitución, la Cobb-Douglas y la translogarítmica.

La función de utilidad del consumidor ecuatoriano considera la supremacía de las tres mercancías en las que más gastan los ciudadanos del país meridional: alimentos y bebidas no alcohólicas, transporte y otros bienes y servicios con una ponderación cercana a la mitad del gasto. Por tipo de zona habitada solo se detecta un cambio importante en el gasto destinado a los alimentos, ya que en las zonas urbanas alcanza una participación de 23 por ciento mientras que en las zonas rurales del 32 por ciento. Además, la zona urbana eleva su gasto en alojamiento, aguas, luz y educación. En el caso del decil I, sin importar la zona en que se habite, los hogares de este nivel socioeconómico destinan 60 por ciento de su ingreso a satisfacer las necesidades básicas, mientras que el decil $X$ utiliza entre el 41 y el 50 por ciento de sus recursos, dependiendo de si habitan zonas urbanas o rurales, respectivamente.

Con base en la presente modelación se puede realizar análisis de los efectos de la aplicación de políticas públicas como el establecimiento de precios controlados, subsidios o de cambios impositivos; este artículo solo es el principio...

\section{Referencias}

Arcarons, J. \& Ramírez, S. (2012). Microeconometría. Madrid: Delta publicaciones.

Cabrer, D., Sancho A. \& Serrano G. (2001). Microeconometría y decisión. Madrid: Ediciones Pirámide.

Cervantes, M. (2014). Microeconomía. Teoria, Simuladores Computacionales y Retos. Ciudad de México: LAES.

Christensen, L., Lau, D. \& Lawrence, J. (1975). Transcendental Logarithmic Utility Functions. The American Economic Review, Vol. 65, No. 3, 367-383.

Ewis, N. \& Fisher, D. (1984). The Translog Utility Function and the Demand for Money in the United States. Journal of Money, Credit and Banking, Vol. 16, No. 1, 34-52.

Fortune, J. (1979). The Translog Utility Function, Quotas, Tariffs, and Consumers. The Translog Utility Function, Quotas, Tariffs, and Consumers, 735743.

Holman, J. (1998). GMM Estimation of a Money-in-the-Utility-Function Model: The Implications of Functional Forms. Journal of Money, Credit and Banking, Vol. 30, No. 4, 679-698.

Instituto Nacional de Estadística y Censos. (2012). Encuesta Nacional de Ingresos y Gastos de los hogares urbanos y rurales 2011- 2012. Resumen metodológico y principales resultados. Ecuador: INEC.

Kim, Y. (1988). The Consumer Demand for Education. The Journal of Human Resources, Vol. 23, No. 2, 173-192. 